\title{
Endometrioma Cyst: To Remove or Not?
}

Pratap Kumar, Anand Balasubramanian

\begin{abstract}
Endometriomas account for $35 \%$ of benign ovarian cyst. Convincing evidence has emerged showing the reduced responsiveness to gonadotropins after ovarian cystectomy. Surgery should be envisaged only in the presence of large cyst or with severe symptoms. For endometriomas less than $3 \mathrm{~cm}$ cyst, aggressive removal of same may lead to a poor response during ovulation inductions.
\end{abstract}

Keywords: Endometrioma, Surgery, Assisted reproduction.

How to cite this article: Kumar P, Balasubramanian A. Endometrioma Cyst: To Remove or Not? Int J Infertility Fetal Med 2012;3(1):26-29.

\section{Source of support: Nil}

Conflict of interest: None declared

\section{INTRODUCTION}

Endometriosis is a disease characterized by ectopic growth of endometrial tissue outside the uterine cavity. Most lesions are located within the peritoneal cavity, involving the peritoneum, ovaries, bowel and pelvic cul-de-sac. Several effective treatments have been reported for infertile women with endometriosis, including controlled ovarian stimulation together with intrauterine insemination, assisted reproductive technology (ART) and surgical therapy. ${ }^{1}$ Endometrioma cyst is one of the most frequent pathologies in gynecologic surgery. The ovary is probably the most common organ affected by endometriosis with ovarian endometriomas occurring in 17 to $44 \%$ of patients with this disease. ${ }^{2}$ Ovarian endometriomas account for $35 \%$ of benign ovarian cysts and are associated with organic type pain, such as chronic pelvic pain and dyspareunia. ${ }^{3}$ There is ongoing debate on how to manage endometriomas, before ART. ${ }^{4}$

\section{REVIEW OF LITERATURE}

Reduced response to controlled ovarian hyperstimulation $(\mathrm{COH})$ after cystectomy has been reported in studies. ${ }^{5}$ Such decreased ovarian reserve might result from surgery ${ }^{6}$ or endometriosis itself. ${ }^{7}$ If at least some of the detrimental effect on ovarian reserve results from the surgery, one would expect more diminished response to $\mathrm{COH}$ after bilateral

Date of Received: 31-03-11

Date of Acceptance: 17-12-11

Date of Publication: January 2012 endometrioma cystectomy as compared to unilateral cystectomy. In a retrospective study, 57 infertile patients who had previously undergone unilateral or bilateral laparoscopic endometrioma cystectomy were analyzed. ${ }^{8}$ The control group consisted of 99 patients with laparoscopically proven tubal factor infertility without hydrosalpinx, endometriosis and prior history of ovarian surgery. All ovarian cystectomies were performed laparoscopically. All patients underwent $\mathrm{COH}$ using luteallong leuprolide acetate and recombinant FSH using the stepdown protocol. The starting dose of gonadotropin was determined based on the woman's age, body mass index (BMI), antral follicle count at baseline transvaginal ultrasonography and day 3 FSH and E2 levels. Ovarian response was monitored with frequent serum E2 measurements and transvaginal ultrasonography. The total FSH level was significantly higher and the peak E2 level was significantly lower in the cystectomy group. The findings of this study suggest that laparoscopic endometrioma cystectomy does reduce the ovarian reserve, as reflected by significantly diminished number of oocytes collected in the operated side as compared to the nonoperated side within the unilateral cystectomy group. Also, requirement of significantly more $\mathrm{FSH}$, production of less oocyte-cumulus complexes and metaphase II oocytes in outcomes of surgical intervention in certain cases of endometriomas, the question that arises is what treatment options are condusive in the management of smaller endometriomas $(<3 \mathrm{~cm})$, and what are the outcomes of IVF/ ICSI on operated and unoperated ovaries. A retrospective study was performed, including 35 women who had undergone endometrioma cystectomy, a total of 38 specimens were studied by three pathologists as three women presented with bilateral localizations, and all cyst diameters measured at least $3 \mathrm{~cm}$. The result of this study showed that endometrioma cystectomy even though performed with an accurate surgical technique leads to significant ovarian tissue removal, the thickness of which increases proportionally with cyst diameter. ${ }^{9}$

The presence of primordial follicles at the cyst capsule may point toward a possible detrimental effect of surgery on ovarian reserve. Study assessed the quantity and nature of ovarian tissue inadvertently resected along with the endometriotic tissue in different parts of the cyst wall, including the cyst adhesion site within the ovarian fossa, 
the intermediate part of the cyst wall and ovarian hilus. It was found that at the initial adhesion site and at the intermediate part of the cyst wall, the ovarian tissue that was removed along with the endometrioma wall mainly constituted of tissue with no follicles or only primordial follicles whereas closer to the ovarian hilus, the ovarian tissue removed along with the endometrioma wall mostly consisted of tissue that contained primary and secondary follicles. ${ }^{4}$

A study was conducted to investigate the effect of conservative surgery of ovarian endometriomas before an intracytoplasmic sperm injection (ICSI) cycle. ${ }^{10}$ Ninety-nine patients with endometriomas who were referred to an ICSI cycle were enrolled in the study. The patients were prospectively randomized into two groups: Group I (49 patients) underwent conservative ovarian surgery before the ICSI cycle and group II (50 patients) underwent the ICSI cycle directly. The stimulation was started 3 months after the operation in group I and directly in group II. In the ovarian surgery group, stimulation was significantly longer (14.0 days in group I and 10.8 days in group II; $p=0.001$ ), total recombinant FSH dose was significantly higher (4575 IU in group I and 3675 IU in group II; $p=0.001$ ) and mean number of mature oocytes was significantly lower (7.8 in group I and 8.6 in group II; $p=0.032$ ). There was no difference in terms of fertilization ( $86 \%$ in group I and $88 \%$ in group II), implantation (16.5\% in group I and $18.5 \%$ in group II) and pregnancy rates (34\% in group I and 38\% in group II). Ovarian surgery resulted in longer stimulation, higher FSH requirement and lower oocyte number, but fertilization, pregnancy and implantation rates did not differ between the groups.

Conflicting information emerging from the literature may be consequent to the fact that endometriomas are mostly monolateral. The contralateral intact ovary may adequately supply for the reduced function of the affected one. To clarify this point, a study assessed IVF-ICSI outcome in women operated on for bilateral endometriomas. Sixty-eight cases and 136 controls were recruited. Women operated on for bilateral endometriotic ovarian cysts had a higher withdrawal rate for poor response $(p<0.001)$. In these patients, despite the use of higher doses of gonadotrophins, the number of follicles $(\mathrm{p}=0.006)$, oocytes retrieved $(p=0.024)$ and embryos obtained $(p=0.024)$ were significantly lower. The study concluded that the IVF outcome is significantly impaired in women operated on for bilateral ovarian endometriomas. ${ }^{11}$

The objective of a retrospective study using 100 endometriosis patients was to establish whether operative treatment of recurrent ovarian endometriosis improves the prognosis of in vitro fertilization. ${ }^{12}$ Forty-five patients had an ovarian endometrioma during IVF treatment, 36 of the cases being recurrences after a previous operation. Fiftyfive patients had ovarian endometriomas operated without recurrence. The patient groups with or without endometriosis did not differ in age, duration of infertility, sperm parameters, amount of gonadotropins required per oocyte and number of retrieved oocytes. The patients with ovarian endometriosis had more embryos (mean 3.9) than women without endometriomas (mean 2.8) $(\mathrm{p}<0.05)$ and the respective pregnancy rates per IVF cycle were 38 and $22 \%$. Patients with endometriomas had a live birth rate of $27 \%$ compared with $20 \%$ in women with no endometriomas. Thus, concluding that the presence of a small endometrioma does not reduce the success of IVF treatment.

A retrospective, matched case-control study, where the objective was to investigate whether conservative surgery on ovarian endometriomas before an IVF cycle improves fertility outcomes. ${ }^{13}$ Around 189 women with endometriomas who underwent IVF treatment: Fifty-six women proceeded directly to IVF, and 133 first underwent conservative ovarian surgery. The interventions used were controlled ovarian hyperstimulation and IVF-ET. The outcome was measured in relation to: Response to gonadotropins, fertilization, implantation and pregnancy rates, the results were as such; aside from lower peak E2 levels on the day of hCG and a higher total FSH dose in women previously operated for an endometrioma, no significant differences were found between the two groups in the different IVF variables analyzed. Hence, concluding that laparoscopic cystectomy for endometriomas before commencing an IVF cycle does not improve fertility outcomes. Proceeding directly to controlled ovarian hyperstimulation in women with asymptomatic ovarian endometriomas might reduce the time to pregnancy, the costs of treatment and the hypothetical complications of laparoscopic surgery.

The purpose of another study was to evaluate the cystectomy-induced damage on the follicular growth and ovulation of an affected ovary during natural cycles. Twenty-eight infertile patients with unilateral ovarian endometriomas who underwent laparoscopic cystectomy were retrospectively evaluated. The ovulation rate of an affected ovary during natural cycles was compared before and after cystectomy in each patient. The results were that after surgery, the ovulation rate was significantly lower than that before cystectomy $(16.9 \pm 4.5 \%$ vs $34.4 \pm 6.6 \%$, $\mathrm{p}=0.013)$. After surgery, 14 pregnancies were achieved without IVF treatment, and only two of them (14.3\%) were achieved from an operated-side ovary. Thus, concluding 
that laparoscopic cystectomy is an invasive treatment in that it reduces the frequency of ovulation; however, the pregnancy rate per ovulation did not deteriorate. ${ }^{14}$

Medical treatment with gonadotropin-releasing hormone analogs improves the outcome of ART. When age, ovarian reserve and male and tubal status permit, surgery should be considered immediately so that time is dedicated to attempt to conceive naturally. In other cases, the preference is for administration of gonadotropinreleasing hormone analogs before ART and no surgery beforehand. The strategy of early surgery, however, seems counterintuitive because of beliefs that milder nonsurgical options should be offered first and surgery last (only if initial treatment attempts fail). Weighing up the relative advantages of surgery, medical treatment and ART are the foundations for a global approach to infertility associated with endometriosis. ${ }^{15}$

Assisted reproductive technology is a widely accepted treatment for infertile women with endometriosis. The presence of an ovarian endometrial cyst reduces the quality of oocytes, while surgical resection of endometrioma may reduce the ovarian reserve for ovarian stimulation by exogenous gonadotropins. A study was done to determine the kind of pretreatment to be given for ovarian endometriotic cyst before IVF-ET. ${ }^{16}$ Infertile women with endometrioma who underwent IVF-ET were divided into three groups in the study as follows:

- Group I: Patients who had received laparotomy or laparoscopy for ovarian endometrioma.

- Group II: Patients for whom the endometrioma content had been aspirated and treated with or without alcohol fixation.

- Group III: Patients who did not undergo pretreatment.

The number of retrieved oocytes, rate of mature oocytes and fertilization rate were compared among groups. The results showed that pretreatment for endometrioma reduces the number of retrieved oocytes. Although oocyte quality as a rate of mature oocytes was not affected by the presence of an ovarian endometrial cyst, the fertilization rate was improved by cyst aspiration. The authors thus propose that surgical pretreatment is not necessary for ovarian endometrial cyst before IVF-ET. However, cyst aspiration has its own disadvantages and is not recommended.

\section{CONCLUSION}

The classic, unproven dogma that ovarian endometrioma should be removed in all infertile women prior to IVF has been recently questioned. Convincing evidence has emerged showing that responsiveness to gonadotrophins after ovarian cystectomy is reduced. Of relevance, here is that surgery exposes women to risk related to a demanding procedure whereas risks associated with expectant management are mostly anecdotal or of doubtful clinical relevance. Surgery should be envisaged only in presence of large cysts (balancing the threshold to operate with the cyst location within the ovary), or to treat concomitant pain symptoms which are refractory to medical treatments, or when malignancy cannot reliably be ruled out. Hence, proceeding directly to IVF to reduce time to pregnancy, to avoid potential surgical complications and to limit patient costs may be useful in women with small endometriomas. For endometriomas lesser than $3 \mathrm{~cm}$, the most plausible treatment option would be to give medical support to the patient along with ART. The presence of a small endometrioma does not reduce the success of ART. One should keep in mind that aggressive removal of endometriotic cyst will remove a large number of oocytes and, hence, the particular individual following ovarian cystectomy becomes a poor responder, particularly when done on both sides.

\section{REFERENCES}

1. Olive DL, Pritts EA. Treatment of endometriosis. N Engl J Med 2001;345:266-75.

2. Jenkins S, Olive DL, Haney AF. Endometriosis: Pathogenetic implications of the anatomic distribution. Obstet Gynecol 1986;67:335-38.

3. Vercellini P. Endometriosis: What pain it is? Semin Reprod Endocrinol 1997;15:251-56.

4. Ibrahim Esinler, Gurkan Bozdag, Funda Aybar, Ulku Bayar, Hakan Yaral. Outcome of in vitro fertilization/intracytoplasmic sperm injection after laparoscopic cystectomy for endometriomas. Fertility and Sterility, June 2006;85(6):1730-34.

5. Ho HY, Lee RK, Hwu YM, Lin MH, Su JT, Tsai YC. Poor response of ovaries with endometrioma previously treated with cystectomy to controlled ovarian hyperstimulation. J Assist Reprod Genet 2002;19:507-11.

6. Suganuma N, Wakahara Y, Ishida D, Asano M, Kitagawa T, Katsumata $Y$, et al. Pretreatment for ovarian endometrial cyst before in vitro fertilization. Gynecol Obstet Invest 2002; 54(Suppl 1):36-42.

7. Bergendal A, Naffah S, Nagy C, Bergqvist A, Sjoblom P, Hillensjo T. Outcome of IVF in patients with endometriosis in comparison with tubal-factor infertility. J Assist Reprod Genet 1998;15:530-34.

8. Bukulmez O, Yarali H, Yucel A, Sari T, Gurgan T. Intracytoplasmic sperm injection versus in vitro fertilization for patients with a tubal factor as their sole cause of infertility: A prospective, randomized trial. Fertil Steril 2000;73:38-42.

9. Roman H, Tarta O, Pura I, Opris I, Bourdel N, Marpeau L, Sabourin JC. Direct proportional relationship between endometrioma size and ovarian parenchyma inadvertently removed during cystectomy, and its implication on the management of enlarged endometriomas. Hum Reprod June 2010;25(6):1428-32. Epub 8 Apr, 2010. 
10. Demirol A, Guven S, Baykal C, Gurgan T. Effect of endometrioma cystectomy on IVF outcome: A prospective randomized study. Reprod Biomed Online May 2006;12(5): 639-43.

11. Somigliana E, Arnoldi M, Benaglia L, Iemmello R, Nicolosi AE, Ragni G. IVF-ICSI outcome in women operated on for bilateral endometriomas. Hum Reprod July 2008;23(7):1526-30. Epub Apr 26, 2008.

12. Tinkanen $H$, Kujansuu E. Acta in vitro fertilization in patients with ovarian endometriomas. Obstet Gynecol Scand Feb 2000;79(2):119-22.

13. Garcia-Velasco JA, Mahutte NG, Corona J, Zúñiga V, Gilés J, Arici A, Pellicer A. Removal of endometriomas before in vitro fertilization does not improve fertility outcomes: A matched, case-control study. Fertil Steril May 2004;81(5):1194-97.

14. Horikawa T, Nakagawa K, Ohgi S, Kojima R, Nakashima A, Ito $\mathrm{M}$, et al. The frequency of ovulation from the affected ovary decreases following laparoscopic cystectomy in infertile women with unilateral endometrioma during a natural cycle. J Assist Reprod Genet June 2008;25(6):239-44.
15. De Ziegler D, Borghese B, Chapron C. Endometriosis and infertility: Pathophysiology and management Lancet Aug 28, 2010;376(9742):730-38.

16. Suganuma N, Wakahara $Y$, Ishida $D$, Asano $M$, Kitagawa $T$, Katsumata Y, et al. Pretreatment for ovarian endometrial cyst before in vitro fertilization. Gynecol Obstet Invest 2002;54 (Suppl 1):36-40; 41-42.

\section{ABOUT THE AUTHORS}

\section{Pratap Kumar (Corresponding Author)}

Professor, Department of Obstetrics and Gynecology, Kasturba Medical College, Manipal University, Manipal, Karnataka, India e-mail: pratap.kumar@manipal.edu

\section{Anand Balasubramanian}

Junior Resident, Department of Obstetrics and Gynecology, Kasturba Medical College, Manipal University, Manipal, Karnataka, India 